found between poor obstetric outcomes and history of thrombosis, presence of SLE or low complement levels (table 1).

Conclusion: In our study, most pregnancies were uneventful. Despite the small sample size, we reinforce the importance of a multidisciplinary evaluation and surveillance before, during and after pregnancy in women with APS in order to implement early treatment and to optimize fetal-maternal outcomes.

Disclosure of Interests: None declared

DOI: 10.1136/annrheumdis-2019-eular.1079

\section{AB0543 1 FEATURES ASSOCIATED WITH RENAL DAMAGE IN PATIENTS WITH SYSTEMIC LUPUS ERYTHEMATOSUS DECEASED OVER A 10-YEAR PERIOD}

Ivan Padjen ${ }^{1}$, Marijan Erceg ${ }^{2}$, Mislav Cerovec ${ }^{1}$, Miroslav Mayer ${ }^{1}$,

Ranko Stevanovic ${ }^{2}$, Branimir Anic ${ }^{1}$. 'University Hospital Centre Zagreb, University of Zagreb School of Medicine, Division of Clinical Immunology and Rheumatology, Department of Internal Medicine, Zagreb, Croatia; ${ }^{2}$ Croatian Institute of Public Health, Zagreb, Croatia

Background: Renal damage (RD) is one of the most important contributors to morbidity and mortality in patients with systemic lupus erythematosus (SLE).

Objectives: We aimed to assess features associated with RD in a group of 90 deceased SLE patients routinely followed-up at our institution, which serves as a national referral center for SLE.

Methods: We retrospectively analyzed 90 SLE patients (68 females) deceased from 2002 to 2011. All patients were $\geq 18$ years of age at death, fulfilling $\geq 4 / 11$ classification criteria of the American College of Rheumatology (ACR). We identified patients with RD, as defined by the Systemic Lupus International Collaborating Clinics (SLICC)/ACR index. An extensive set of variables was compared between patients with and without RD (RD and RD-N, respectively): demographics, ACR criteria at diagnosis and cumulatively at death, total damage and its components one year following diagnosis and non-renal damage and its components cumulatively at death, as well as components of the metabolic syndrome, smoking, sicca and Hughes syndrome. Frequencies were compared using the chi-square and Fisher's exact test, and continuous variables using the t-test and Mann-Whitney $U$ test. Variables associated with RD were analyzed using multivariate logistic regression.

Results: We identified 25/90 patients who accrued RD over the course of their disease. In the univariate analysis, we found no difference between $\mathrm{RD}$ and RD-N patients in any of the following parameters: demographics, total count of ACR criteria at diagnosis and death, as well as damage at one year after diagnosis and cumulative non-renal damage at death. Compared to RD-N patients, RD patients had a higher proportion of malar rash at diagnosis $(11 / 25$ vs. $13 / 65, p=0.021)$ and a higher cumulative proportion of renal disorder (19/25 vs. $30 / 65, p=0.011)$, including proteinuria and urinary casts (17/25 vs. 23/65, $p=0.005$, for both). RD patients also had a higher proportion of myocardial infarction as an item of cumulative damage $(7 / 25$ vs. $6 / 65, p=0.023)$ and were more frequently obese (11/25 vs. 15/65, $\mathrm{p}=0.049)$. Conversely, hematological disorder and leukopenia at diagnosis were less frequent in RD compared to RD-N patients (4/25 vs. $30 / 65, \quad \mathrm{p}=0.008$ and $1 / 25$ vs. $21 / 65$, respectively). In the final multivariate model (adjusted for gender, age at diagnosis and disease duration), malar rash at diagnosis and the cumulative presence of renal disorder (classification criteria of the ACR) were positively associated with RD. Conversely, leukopenia at diagnosis was inversely associated with RD (Figure 1).

\begin{tabular}{lccc} 
Variable & OR & $95 \% \mathrm{Cl}$ & $\mathrm{p}$ \\
\hline Malar rash (dg)(yes/no) & $\mathbf{2 7 . 4 1 6}$ & $\mathbf{3 . 5 8 6 - 2 0 9 . 6 2 0}$ & $\mathbf{0 . 0 0 1}$ \\
Leukopenia (dg)(yes/no) & $\mathbf{0 . 0 1 6}$ & $\mathbf{0 . 0 0 1 - 0 . 2 7 3}$ & $\mathbf{0 . 0 0 4}$ \\
Renal disorder (cum)(yes/no) & $\mathbf{7 . 1 4 1}$ & $\mathbf{1 . 0 9 1 - 4 6 . 7 6 0}$ & $\mathbf{0 . 0 4 0}$ \\
Myocardial infarction (cum)(yes/no) & 6.117 & $0.319-117.197$ & 0.229 \\
Obesity (cum)(yes/no) & 1.917 & $0.425-8.638$ & 0.397 \\
Male gender (yes/no) & 1.913 & $0.335-10.916$ & 0.465 \\
Age (dg)(years, cont) & 1.018 & $0.969-1.070$ & 0.481 \\
Disease duration (cum)(years, cont) & 0.940 & $0.826-1.070$ & 0.352 \\
\hline
\end{tabular}

$\mathrm{dg}$ - at diagnosis, cum - cumulatively, yes/no - binary variable, cont - continuous variable

Figure 1. Features associated with renal damage (multivariate logistic regression model)
Conclusion: More than a quarter of deceased patients accrued RD. While malar rash at diagnosis may be associated with a higher likelihood of developing RD, early leukopenia may be associated with its lower likelihood in deceased patients.

\section{REFERENCES}

[1] Davidson A. Nat Rev Rheumatol. 2016;12:143-53.

Disclosure of Interests: Ivan Padjen: None declared, Marijan Erceg: None declared, Mislav Cerovec: None declared, Miroslav Mayer Speakers bureau: Novartis, Sandoz, Abbvie, Pfizer, Alvogen, Roche, MSD, Octapharma, Ranko Stevanovic: None declared, Branimir Anic Speakers bureau: Novartis, Sandoz, Abbvie, Pfizer, Alvogen, Roche, MSD Octapharma

DOI: 10.1136/annrheumdis-2019-eular.2885

\section{AB0544 ECHOCARDIOGRAPHIC CHANGES IN PATIENTS WITH SYSTEMIC LUPUS ERYTHEMATOSUS BEFORE TO INITIATION OF IMMUNOSUPPRESSIVE THERAPY}

Tatiana Panafidina ${ }^{1}$, Tatiana Popkova ${ }^{2}$, Alexander Volkov ${ }^{3} .{ }^{1}$ V.A.Nasonova Research Institute of Rheumatology, Systemic rheumatic diseases, Moscow, Russian Federation; ${ }^{1}$ V.A.Nasonova Research Institute of Rheumatology, Systemic rheumatic diseases, Moscow, Russian Federation; ${ }^{3}$ V.A. Nasonova Research Institute of Rheumatology, Diagnostic department, Moscow, Russian Federation

Background: Cardiovascular diseases are becoming the leading cause of death among SLE patients due to increasing life-spans. Transthoracic echocardiography (TTE) is a routine and widely available modality in everyday clinical practice useful to identify specific pathological cardiac changes and predictors of heart failure (HF).

Objectives: Obtaining of specific TTE findings in SLE patients prior to initiation of pathogenic immunosuppressive therapy was the objective in this study. Methods: Thirty four pts (91\% females, aged 30[26-34]years (median [interquartile range 25\%-75\%]) with "non-treated" SLE (ACR 1997 and SLICC 2012 criteria) were included. None of pts was treated either with prednisone or cytotoxic drugs at the moment of inclusion.

Results: Median SLE duration was 18[6-60]months, SLEDAI-2K - 13[8-19], SLICC/DI - 0[0-0]scores. Leading SLE clinical manifestations included: hematological changes $(74 \%)$, kidney involvement $(59 \%)$, joints $(50 \%)$ and skin involvement $(50 \%)$. Immunological abnormalities were detected in all patients and were as follows: ANA positivity - in 100\%, anti-dsDNA antibodies - in $76 \%$ of SLE patients. Concurrent antiphospholipid syndrome was found in $2(6 \%)$ patients. Valve insufficiency with varying degree of regurgitation was the commonest pathology found in "non-treated" SLE patients based on TTE data: mitral valve insufficiency - in 31(91\%), tricuspid valve - in $31(91 \%)$, pulmonary valve insufficiency - in $21(62 \%)$, aortic valve insufficiency - in $4(12 \%)$ patients. Endocarditis was a rare pathology found in $5(15 \%)$ patients, while mitral and tricuspid valves prolapse was seen more often - in 16(47\%), while not a single case of valve stenosis was found. Pericardial pathology was detected in $16(47 \%)$ patients: exudative - in $9(26 \%)$, and adhesive (thickening, hardening and separation of leaflets) - in $7(21 \%)$

There were no cases of CAD or $\mathrm{Ml}$, although there were $2(6 \%)$ documented cases of cerebral stroke in past history, and $1(3 \%)$ case of confirmed CHF. Most common TRF were dyslipidemia and hypertension - in $15(44 \%)$ and $11(32 \%)$ SLE patients respectively.

Median LVEF was 64[59-67]\%, LV end-systolic dimension- 30[27-32]mm, LV end-diastolic dimension - 48[45-51]mm, pulmonary artery systolic pressure - 24[22-32] $\mathrm{mm} \mathrm{Hg}$. LV diastolic disfunction (LVDD) was found in 10 (29\%), systolic dysfunction (LVSD) - in 4(12\%), LV myocardial hypertrophy $(\mathrm{LVH})$ - in $5(15 \%)$; left atrium dilatation (LAD) was found in $4(12 \%)$, and increased dimensions of right atrium was detected in 3(9\%) SLE patients.

Conclusion: Most common cardiac abnormalities in "non-treated" SLE patients with high activity (SLEDAI-2K 13 scores) were valve dysfunction (insufficiency with regurgitation), mitral and tricuspid valve prolapse and pericarditis. Of importance is the presence of early subclinical features of HF almost in $1 / 3$ of naïve to treatment SLE patients: LVDD (29\%), LVH $(15 \%)$, and LAD (12\%). SLE patients should be thoroughly monitored both for adequate control of SLE activity, and cardiac pathology with correction of TRF, regular assessments by a cardiologist, TTE, and early administration of cardio-protection therapy in view of increased HF risk in SLE patients, predetermining unfavorable prognosis.

Disclosure of Interests: None declared

DOI: 10.1136/annrheumdis-2019-eular.7094 